\title{
EXPERIENCIA DEL PROCESO DE MODELACIÓN MATEMÁTICA COMO ESTRATEGIA DIDÁCTICA EN LA ENSEÑANZA DE LA MATEMÁTICA FINANCIERA
}

\author{
Emiliano Grueso Cárdenas, Liliana Potosí Cruz y Sandra Esther Suárez \\ Grupo de investigación en Simulación y Ciencias Básicas Aplicadas (GISCBA) \\ Institución Universitaria Antonio José Camacho \\ Recibido: 03/03/2020. Aprobado: 27/07/2020 \\ Cómo citar este artículo: \\ Grueso Cárdenas, E., Potosí Cruz, L. y Suárez, S.A. (2020). Experiencia del proceso de modelación matemática como \\ estrategia didáctica en la enseñanza de la matemática financiera. Revista Sapientía, 12(24), 16-26.
}

\section{RESUMEN}

El objetivo de este artículo es dar a conocer el desarrollo de una experiencia de investigación basada en el proceso de modelación matemática como estrategia didáctica del aprendizaje en matemática financiera. Una vez identificado el problema que presentan las personas en el proceso de formación financiera al emplear las herramientas de matemática básica, se evidencia la dificultad para hacer transposiciones didácticas entre el concepto financiero y las relaciones matemáticas que este contempla. Los hallazgos del estudio se obtuvieron a partir de una triangulación metodológica, donde los métodos cuantitativo y cualitativo permitieron observar algunas necesidades académicas de los estudiantes de tercer semestre de la Facultad de Ciencias Empresariales de la Institución universitaria Antonio José Camacho (UNIAJC), en el primer periodo del 2017, las cuales fueron el derrotero para plantear una Guía de trabajo integral que une los conceptos básicos de matemáticas requeridos en temas de matemática financiera. Los resultados del proceso descritos en esta experiencia, mostraron un impacto positivo tras la aplicación de la Guía en los cursos de matemática financiera.

\section{PALABRAS CLAVE}

Matemática Financiera, Modelación, TIC.

\section{ABSTRACT}

The objective of this article is to present the development of a research experience based on the process of mathematical modeling as a didactic strategy of learning in financial mathematics. Once the problem that people present in the financial training process when using the basic mathematics tools has been identified, the difficulty in making didactic transpositions between the financial concept and the mathematical relationships that it contemplates becomes evident. The study findings were obtained from a methodological triangulation, where the quantitative and qualitative methods allowed to observe some academic needs of the students of the third semester of the Faculty of Business Sciences of the Antonio José Camacho University Institution 
(UNIAJC), in the first period of 2017, which were the path to plant a comprehensive Work Guide that unites the basic concepts of mathematics required in financial mathematics. The results of the process in this experience, problems a positive impact after the application of the Guide in financial mathematics courses

\section{KEYWORDS}

Financial Mathematics, Modeling, TIC.

\section{INTRODUCCIÓN}

Las exigencias del mundo globalizado contemplan diversos aspectos, entre ellos el análisis del impacto educativo nacional respecto de los conocimientos que presenta el estudiante en su educación financiera. Dichos conocimientos son evaluados por la Organización para la Cooperación y el Desarrollo Económico (OCDE) mediante pruebas internacionales como las del Programme for International Student Assessment (PISA), en las que se analiza el nivel del alfabetismo financiero, definido como:

El conocimiento y comprensión de conceptos financieros y del riesgo, de las habilidades, actitud y confianza para la aplicación de estos conocimientos en la toma de decisiones efectivas en contextos financieros, para mejorar el bienestar financiero de los individuos y la sociedad, y para permitir la participación de los individuos en la economía (OECD, 2017).

Tras los alarmantes resultados de Colombia en la Prueba PISA aplicada en 2012, el gobierno colombiano trabajó posteriormente en un documento llamado Orientaciones Pedagógicas para la Educación Económica y Financiera a cargo del MEN, instaurando pautas generales para la implementación transversal en el plan de estudio ofrecido a los estudiantes por las instituciones educativas y poder de esta manera responder positivamente en los resultados de la prueba del 2015.
Pese a estos intentos, Aguirre (2015) menciona que:

En la prueba PISA aplicada en el año 2015 a estudiantes cuyas edades eran de 15 años, los estudiantes colombianos mostraron ser los últimos en competencias de Matemática Financiera. Los jóvenes obtuvieron 379 puntos, los cuales los ubicó en el último puesto y china que obtuvo el primer lugar con 603 puntos, Bélgica con 503 puntos, Estonia con 529 puntos, Austria 526 puntos, Nueva Zelanda 520 puntos, República Checa 513 puntos, Polonia 510 y Letonia 501 puntos. Se evaluaron 29.000 escolares de 18 naciones, los temas fueron manejo de cuentas y tarjetas bancarias, proyección de sus finanzas, intereses y sus derechos y deberes como ciudadanos (p.10).

En este sentido, cuando se trabaja Matemática Financiera en los grupos de estudiantes de la UNIAJC, un porcentaje entre el $88 \%$ y $90 \%$ responde no haber recibido fundamentos de educación financiera en las instituciones educativas donde cursaron estudios de primaria o bachillerato, según Grueso (2017). Esto da fe de un analfabetismo en el ámbito económico y financiero.

La no educación financiera en los primeros años de escolaridad no es la única causa del analfabetismo financiero. Cuando a lo financiero se le antecede la palabra matemática la mayoría de los estudiantes se previenen, creando una barrera por la experiencia que han tenido con las matemáticas básicas. Tal como lo menciona Edel y García (2009), quienes describen esta situación como una "complicación en el proceso de enseñanza y aprendizaje del curso, aversión, desinterés, materia que genera dudas constantes. Fenómeno que se viene presentando históricamente desde la creación del concepto matemático" (p.2).

Aunque los estudiantes antes de tomar el curso de matemática financiera han aprobado dos cursos de matemáticas básicas, presentan dificultad en la comprensión y la significación de las relaciones y operaciones matemáticas que están inmersas en el ámbito financiero, lo que se convierte en un obstáculo para el proceso de modelación. 
Por lo anterior, el objeto de este artículo es dar a conocer la experiencia de investigación basada en el proceso de modelación matemática como estrategia didáctica del aprendizaje en matemática financiera, con el fin de que puedan ser aplicados en los cursos de matemática financiera y de esta manera mitigar las falencias con las que llegan los estudiantes para iniciar su proceso de formación financiera.

Dentro del desarrollo de la experiencia se plantea un apartado de hallazgos, donde se consideran los resultados socio cognitivos de los estudiantes de tercer semestre de Facultad de Ciencias Empresariales de la UNIAJC, identificando algunas dificultades en conceptos matemáticos básicos y financieros, las cuales afectan notablemente el desarrollo del proceso de la modelación en la matemática financiera.

Posteriormente, se plantean un aparte de resultados, donde se da un ejemplo de la manera como se aborda una temática financiera empleando el proceso de la modelación que permita satisfacer necesidades de matemática básica con las que llega el estudiante, el cual responde a una "Guía" didáctica, que le permite al estudiante afianzar el proceso de modelación de la matemática, participar y cooperar de manera activa en la apropiación de elementos matemáticos que faciliten la modelación en el ámbito financiero como método de enseñanza aprendizaje (Alaniz et. al, 2006).

\section{MARCO TEÓRICO}

Los conceptos de Modelación y Modelización tienen definiciones diferentes en la actualidad, sin embargo, históricamente en Matemáticas la Modelación estaba definida como hoy se define la Modelización. Biembengut y Hein (2000) definen el proceso de modelación matemática como una aplicación de la modelización en la enseñanza aprendizaje.

Por su parte, Villa (2007) establece una diferencia entre los procesos de Modelización y Modelación en el campo de las matemáticas: la primera la asume como el acto de una actividad científica y segunda como una herramienta en el aula de clase (Villa, 2007, citado en Grueso, 2017, p.13). La tabla a continuación facilita reconocer, ampliar y establecer diferencias.

Tabla 1. Algunas diferencias entre los procesos de Modelización y de Modelación en el campo de las Matemáticas

\begin{tabular}{|c|c|c|}
\hline Criterio & $\begin{array}{l}\text { Como actividad } \\
\text { científica }\end{array}$ & $\begin{array}{l}\text { Como herramienta } \\
\text { en el aula de clase }\end{array}$ \\
\hline $\begin{array}{l}\text { Propósito del } \\
\text { modelo }\end{array}$ & $\begin{array}{l}\text { El modelo se } \\
\text { construye para } \\
\text { solucionar un } \\
\text { problema de } \\
\text { otras ciencias } \\
\text { (naturales, sociales, } \\
\text { humanas...) o para } \\
\text { avanzar en una } \\
\text { teoría o ciencia. }\end{array}$ & $\begin{array}{l}\text { El modelo se elabora } \\
\text { para construir } \\
\text { un concepto } \\
\text { matemático dotado } \\
\text { de un significado } \\
\text { y con la intención } \\
\text { de despertar una } \\
\text { motivación e interés } \\
\text { por las matemáticas } \\
\text { debido a su carácter } \\
\text { aplicativo. }\end{array}$ \\
\hline $\begin{array}{l}\text { Los conceptos } \\
\text { matemáticos }\end{array}$ & $\begin{array}{l}\text { Emergen de la } \\
\text { situación a través } \\
\text { de un proceso } \\
\text { de abstracción y } \\
\text { simplificación del } \\
\text { fenómeno. }\end{array}$ & $\begin{array}{l}\text { Deben haber sido } \\
\text { considerados a priori } \\
\text { con base en la prepa- } \\
\text { ración y selección del } \\
\text { contexto por parte } \\
\text { del maestro y de } \\
\text { acuerdo con los pro- } \\
\text { pósitos de la clase. }\end{array}$ \\
\hline Contextos & $\begin{array}{l}\text { Obedece a } \\
\text { problemas que } \\
\text { comúnmente no } \\
\text { han sido abordados } \\
\text { o se abordan de una } \\
\text { manera diferente } \\
\text { al interior de la } \\
\text { ciencia. }\end{array}$ & $\begin{array}{l}\text { Deben obedecer a } \\
\text { problemas abordados } \\
\text { previamente } \\
\text { por el docente } \\
\text { de la clase con el } \\
\text { objeto de evaluar } \\
\text { su pertinencia } \\
\text { con propósitos } \\
\text { educativos. }\end{array}$ \\
\hline Otros factores & $\begin{array}{l}\text { Se presenta } \\
\text { generalmente en un } \\
\text { ambiente propio de } \\
\text { la ciencia en la cual se } \\
\text { aplica y generalmente } \\
\text { es externo a factores } \\
\text { educativos. }\end{array}$ & $\begin{array}{l}\text { Se presenta } \\
\text { regularmente en el } \\
\text { aula de clase bajo } \\
\text { una motivación } \\
\text { propia de contextos } \\
\text { cotidianos y de } \\
\text { otras ciencias. }\end{array}$ \\
\hline
\end{tabular}

Fuente. La modelación como proceso en el aula de matemáticas: Un marco de referencia y un ejemplo (Villa, 2007 citado en Grueso, 2017, p.13). 
Las Matemáticas en su estructura de enseñanzaaprendizaje contemplan cinco procesos, entre ellos la Modelación, definida por el holandés Hans Freundenthal como aquella que integra una situación problemática o real, la cual debe ser planteada matemáticamente, llegando a un modelo donde exista coherencia interna entre la situación problemática, la formulación del problema y el modelo, validándolo mediante predicciones que satisfagan todas las etapas del proceso (MEN Colombia,1998, p.76). Este proceso es de gran utilidad, pero los estudiantes presentan dificultad para usarlo debido a obstáculos epistemológicos y didácticos (D’Amore, 2006).

El trabajo didáctico del docente en el proceso de formación del estudiante, haciendo entrega de una fórmula y que empiece a resolver situaciones que se asemejen a ella como etapa inicial, no es suficiente para que el estudiante logre hacer transposiciones didácticas a otros contextos. El contexto de la matemática financiera es un buen referente de trabajo para implementar el proceso de modelación, ya que los estudiantes ven la necesidad de tener educación financiera, que se debería trabajar desde los primeros años de escolaridad según la respuesta a una de las preguntas de la encuesta sociodemográfica aplicada.

El enfatizar con el proceso de la modelación matemática como elemento didáctico en contextos financieros y llevarlo a la hoja de cálculo Excel minimiza la no apropiación de saberes por parte del estudiante en su educación financiera, lo cual se verá reflejado en sus interacciones socioeconómicas.

El uso de la Tecnología de la Información y la Comunicación (TIC) es una herramienta de gran importancia para el proceso de modelación, que facilita al estudiante agilizar los cálculos dando respuestas, así como inferir otros posibles resultados.

\section{METODOLOGÍA}

La investigación se realizó empleando un diseño metodológico basado en un método mixto, que combina el método cualitativo y cuantitativo, donde se integraron las respuestas de tres instrumentos aplicados a los estudiantes como la encuesta, el diagnóstico y una entrevista. En este orden, se observaron algunas características sociodemográficas, elementos básicos de matemáticas que inciden en la no educación financiera de las personas y, por último, el reconocimiento del impacto de la propuesta de trabajo.

Las preguntas realizadas y las respuestas dadas por los estudiantes en la encuesta y el diagnóstico llevó a los educandos cuestionarse sobre algunos elementos sociales y académicos que inciden notoriamente en su proceso de formación, los cuales se tuvieron presentes para organizar la creación de la propuesta Guía, contemplando el fortalecimiento del pensamiento lógico matemático desde el pensamiento numérico y el pensamiento variacional a través del proceso de la modelación matemática en un contexto financiero.

Finalmente, se utilizó la entrevista como un indicador para conocer el alcance de la Guía de trabajo frente a la asimilación del curso de matemática financiera por parte de los estudiantes de acuerdo con los temas programados en ella.

La muestra de esta investigación se consolidó con dos grupos de estudiantes de la UNIAJC de tercer semestre, periodo 2017-1, uno de Tecnología en Gestión Empresarial (355) de jornada matinal y el otro Profesional en Administración (3155) de jornada nocturna. Los grupos en mención están bajo el mismo micro currículo de las asignaturas: matemática básica y matemática financiera (Grueso, 2017, p.20).

\section{HALLAZGOS}

La modelación requiere que el estudiante tenga dominio de elementos básicos y fundamentales de la matemática para poder modelar una situación en contexto. Al respecto, el diagnóstico aplicado a estudiantes de ciencias empresariales evidenció problemas al identificar los numerales que representan números reales y su interpretación de acuerdo con el 
contexto financiero. Otro aspecto en el que presentan dificultades es en las propiedades de números reales para despejar una variable o incógnita.

$\mathrm{Al}$ analizar la encuesta de percepción se encontró lo siguiente:

G 355 ¿recibió conceptos de matemática financiera en sus Estudios de primaria y/o bachillerato?

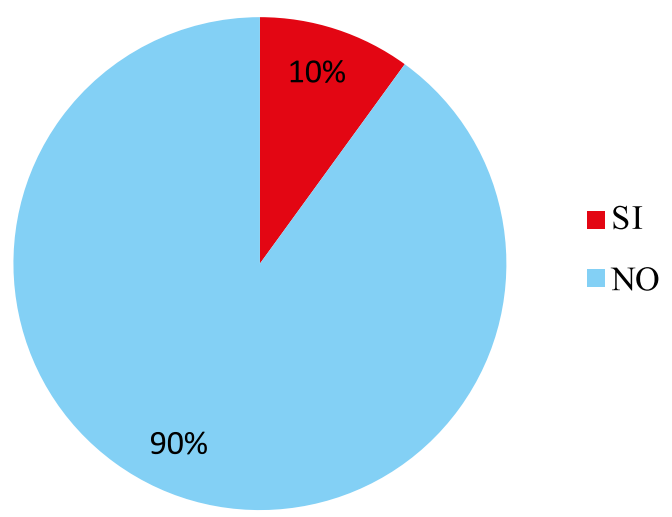

Figura 1. Conceptos de Matemática Financiera recibidos por los estudiantes del grupo 355 en sus estudios de primaria o bachillerato. Fuente: Grueso, E. (2017).

Grupo 3155 ¿recibió conceptos de matemática financiera en sus estudios de primaria y/o bachillerato?

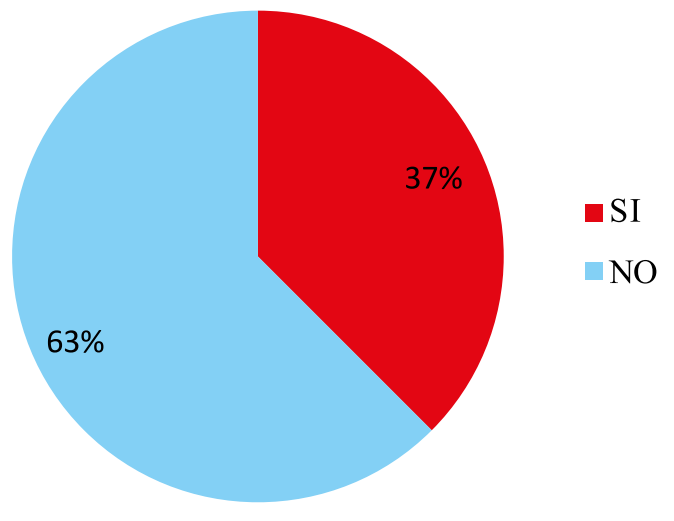

Figura 2. Conceptos de Matemática Financiera recibidos por los estudiantes del grupo 3155 en sus estudios de primaria o bachillerato.

Fuente: Grueso, E. (2017).

En la consulta a los estudiantes sobre la adquisición de elementos de matemática financiera a lo largo de sus estudios de la básica y la media, un porcentaje inferior al 24\% respondió afirmativamente, sin embargo, sus respuestas no estaban relacionadas directamente con la pregunta, ya que la asociaban con temas de materias de estadística descriptiva y contabilidad. El resto manifestaron no haber recibido formación financiera, lo cual queda como una situación problema en lo que concierne al faltante con el que llegan los estudiantes a la universidad en esta área. (Ver figuras 1y2).

Grupo 355 ¿es importante la formación financiera en un estudiante?

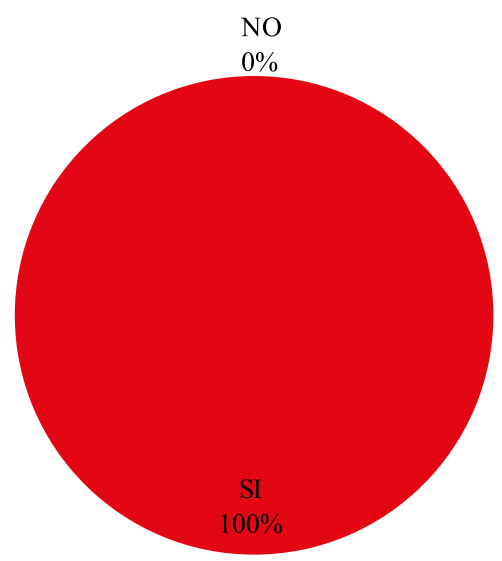

Figura 3. Percepción de los estudiantes del grupo 355 frente a la importancia de la formación financiera.

Fuente: Grueso, E. (2017).

G3155 ¿es importante la formación financiera en un estudiante?

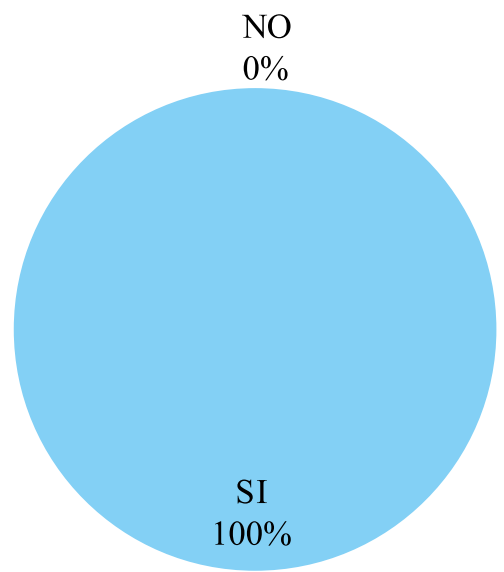

Figura 4. Percepción de los estudiantes del grupo 3155 frente a la importancia de la formación financiera.

Fuente: Grueso, E. (2017). 
En las Figuras 3 y 4 las respuestas de los estudiantes llaman la atención porque dejan ver una postura con tendencia absoluta, lo que puede obedecer a las interacciones en el diario vivir en sus contextos económicos y sociales, para afrontarlos de manera organizada y planeada a fin de obtener mejores resultados y bienestar, reconociendo por tal motivo la importancia de la formación financiera.

\section{G 355 ¿qué herramientas tecnológicas le sirven de apoyo} en la formación de la matemática financiera?

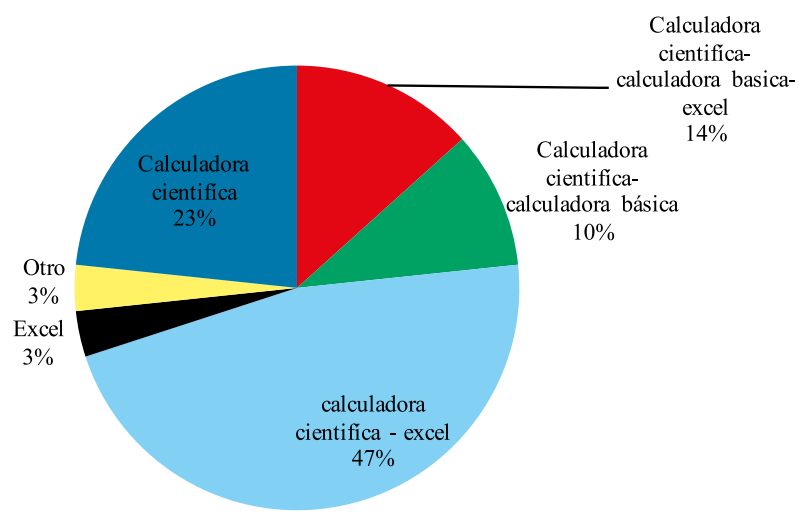

Figura 5. Herramientas tecnológicas empleadas por el grupo 355 que sirven de apoyo en la formación de Matemática Financiera.

Fuente: Grueso, E. (2017).

Grupo 3155 ¿qué herramientas tecnológica le sirven de apoyo en la formación de la matemática financiera

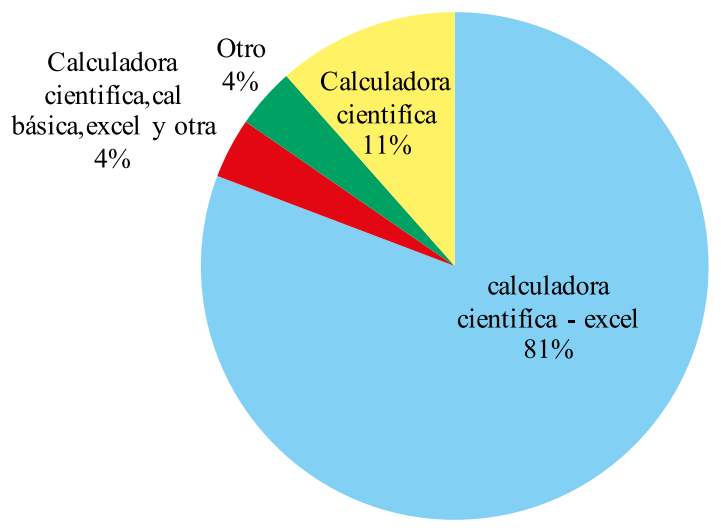

Figura 6. Herramientas tecnológicas empleadas por el grupo 3155 que sirven de apoyo en la formación de Matemática Financiera.

Fuente: Grueso, E. (2017).
Los estudiantes dejan ver en sus respuestas lo significativo que es el uso de la calculadora científica al igual que la hoja de Excel para el trabajo de los cálculos en matemática financiera. Estos dos elementos guardan estrecha relación con el proceso de modelación que los estudiantes van adquiriendo, lo que coadyuva en la claridad conceptual para procesar los datos, fortaleciendo la propuesta de investigación (ver figura 5 y 6).

Al analizar la prueba diagnóstica aplicada se encontró lo siguiente:

Pregunta 1. Opere las siguientes expresiones y observe si son equivalentes o diferentes.

a. Cuánto dinero representan de \$1'000.000

b. Cuánto dinero representa 0.25 partes de $\$ 1^{\prime} 000.000$.

c. La cuarta parte de un millón.

d. Calcular el $25 \%$ de $\$ 500.000$ más $25 \%$ de quinientos mil pesos.

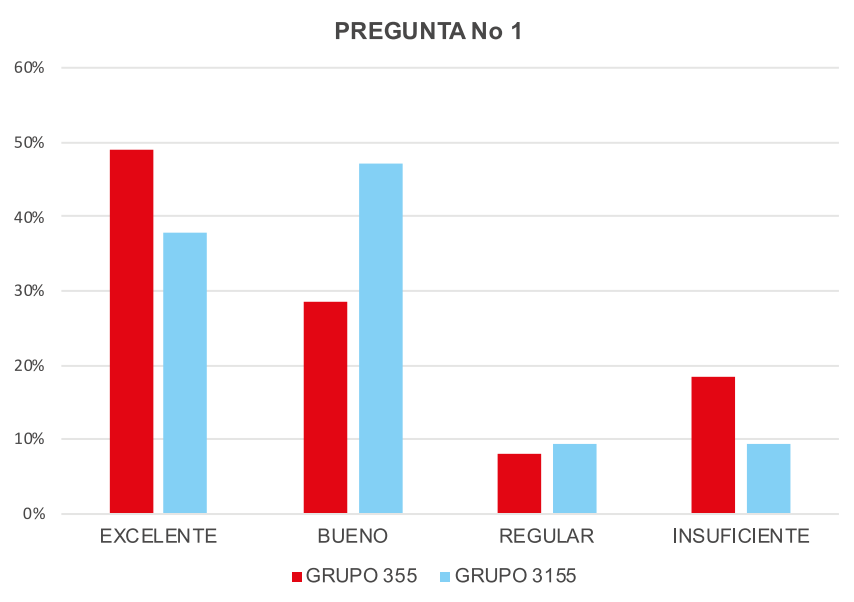

Figura 7. Respuesta a la pregunta No 01 del diagnóstico. Representaciones equivalentes de un número, para cálculo de porcentaje.

Fuente: Grueso, E. (2017).

Pese a que el estudiante debe de venir con formación básica que le permita reconocer las 
representaciones de equivalencia para trabajar la razón porcentual de una cantidad, solo el $43 \%$ de los diagnosticados reconocen que existen diferentes formas de presentar el mismo número real, el cual cobra un sentido de interpretación acorde al contexto trabajado y, en especial, al cálculo de los porcentajes, tema requerido de manera frecuente en matemática financiera.

Pregunta 2. Hallar el valor de x e y de las ecuaciones:

$$
\text { a. } 4 x+5=0 \text { b. } \frac{y}{5}+2 y-\frac{4}{3}=2
$$

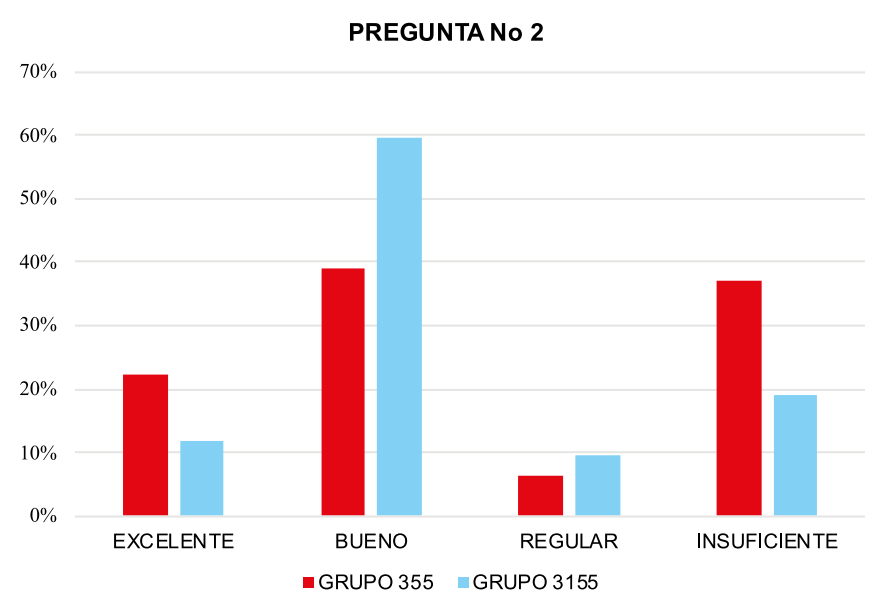

Figura 8. Respuesta a la pregunta No 02 del diagnóstico. Aplicación de propiedades de los reales en el despeje variables o incógnitas.

Fuente: Grueso, E. (2017).

La matemática financiera requiere de elementos fundamentales propios de la matemática como la aplicación de propiedades de las operaciones con números reales en el despeje de una variable. Menos del $16 \%$ de los estudiantes efectúa correctamente esta operación, siendo este resultado muy bajo con relación al requerimiento básico necesario para el proceso de formación en matemática financiera, según Carazo y Brey (2012).

Pregunta 3. Hallar el valor de t en años, justifique su respuesta a. ${ }^{100^{t}}=10 \mathrm{~b}$. Exprese a t en términos de $\mathbf{x}(x+5)^{t}=100$

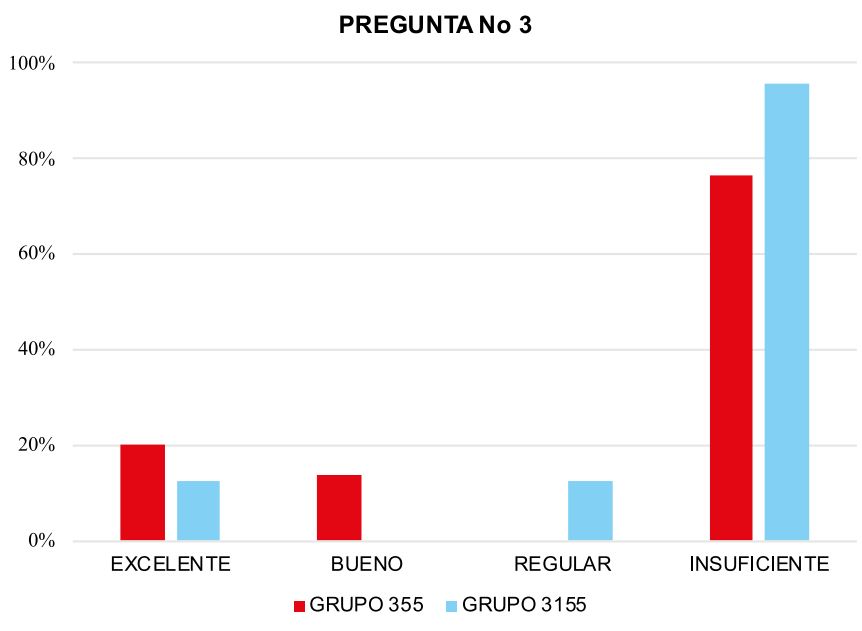

Figura 9. Respuesta a la pregunta No 06 del diagnóstico. Identificar el concepto de exponencial, logaritmo y propiedades para despejar una variable que aparezca como exponente.

Fuente: Grueso, E. (2017).

La figura 9 muestra un desconocimiento de la mayoría de los estudiantes diagnosticados en relación con el despeje de una variable que se encuentra como exponente, operación que se encuentra en situaciones de interés compuesto, amortizaciones y capitalizaciones, donde el tiempo está como exponente. De manera que, al momento de hallar dichos cálculos, al estudiante se encontrará con una dificultad.

En este sentido, se evidencia que los estudiantes están muy poco familiarizados con el tema de ecuación exponencial y logarítmica y, en especial, con el despeje de incógnitas. Situación que, como señalan Carazo y Brey (2012), se convierte en obstáculo para trabajar procesos de modelación en matemática financiera.

El análisis de las respuestas dadas por los estudiantes en la encuesta y el diagnóstico avalan la fundamentación de la estrategia didáctica que propone la investigación y mediante la cual se logra minimizar las dificultades de algunos procesos matemáticos en la aplicación de propiedades de números reales, usándolos en la modelación matemática del contexto financiero y permitiendo 
a los estudiantes alcanzar una formación financiera que impacte de manera positiva su diario vivir.

\section{RESULTADOS}

Tras los hallazgos obtenidos mediante la metodología de triangulación, representada en las falencias matemáticas básicas da como resultado proponer una estrategia didáctica a través de un documento que se le llamó GUIA, en la cual se preserva un proceso didáctico pedagógico donde el docente y el estudiante interactúan para lograr el propósito de la comprensión de un tema del ámbito financiero.

La estructura de la guía, abarca los temas de porcentaje, interés simple, interés compuesto $\mathrm{y}$ anualidades, las cuales se trabajan tema por modulo bajo el siguiente esquema:

Tema: Es el elemento fundamental de impacto social donde se ve inmerso el estudiante a la luz de su formación personal con relación directa en competencias de su plan de estudios.

\section{Módulo 2/ Tema: Interés Simple}

Introducción: Se busca con ella, el interactuar del docente y el estudiante para elevar más la motivación hacia el derrotero que emprenden a través de una pregunta en metodología mayéutica.

Módulo 2/ Introducción: Las personas diariamente se ven implicadas en el uso del dinero, donde puede recibir a favor o pagar, dependiendo de la postura que asuma en la transacción. El conocer o tener educación financiera permite optimizar el uso del dinero que se recibe o se paga.

Competencia: Considera el alcance cognitivo que desarrolla el estudiante en la apropiación del concepto financiero.

Módulo 2/ Competencia: Tener apropiación y dominio en reconocer, y aplicar el Interés Simple, generando seguridad para la toma de decisiones en contextos comerciales y financieros.

Conceptos Básicos: Hacen referencia a elementos matemáticos fundamentales, que servirán de apoyo en el proceso de la modelación en contexto. Módulo 2/ Conceptos básicos: Adición, sustracción, multiplicación y división con los números reales.

Modelación: Proceso matemático llevado al aula de clase con la estructura dada por Hans Freundenthal, donde a los estudiantes se les da una situación real del ámbito financiero para establecer las relaciones matemáticas inmersas en él, lo cual conlleva a una formulación de la situación a través de las representaciones semióticas como plantea Duval, lo que en conjunto con las operaciones matemáticas generan el Modelo. El objetivo de la sección de Modelación dentro de cada módulo por tema es que el estudiante logre mediante este proceso un aprendizaje significativo del cual se pueden transponer en otras representaciones financieras y a otros campos de aplicación.

Módulo 2/ Modelación: Cuando se da el concepto de Interés, se identifican los elementos que se relacionan a través de operaciones matemáticas. Al expresar el Interés como el pago en dinero por el uso del dinero a lo largo de un periodo de tiempo, el concepto habla de dos momentos del dinero, el dinero en el presente y el dinero al pasar el tiempo, son dos elementos que se relacionan bajo la operación sustracción que semióticamente se puede representar de la siguiente manera:

\section{I: Interés}

\section{$\mathrm{N}$ : Dinero en el presente}

\section{J: Dinero en el futuro.}

Como el Interés ganado es la diferencia entre el dinero en el futuro y el dinero en el presente se puede modelar como $\mathrm{I}=\mathrm{J}-\mathrm{N}$, que con las situaciones reales que se presentan en el módulo 
el estudiante visualiza con mayor facilidad para acceder al proceso de la modelación.

Cuando se presenta el concepto de Tasa de Interés desde el ámbito financiero se puede ver como la razón entre los intereses y el producto del dinero inicial en el tiempo. Semióticamente, la tasa de interés, se representa por la letra $i$, el tiempo por la t, el valor inicial presente con la letra "N" y los intereses ganados en el tiempo con la letra "I". La relación de la operación razón está movilizada matemáticamente por la operación división, logrando integrar las partes de la siguiente manera $\mathrm{i}=\mathrm{I} / \mathrm{N}^{\star} \mathrm{t}$.

\section{SIMULACIÓN}

Finalmente, en cada módulo se propone al estudiante llevar el modelo construido a la hoja de Cálculo Excel; para que aplicando las herramientas TIC's amplié el análisis para la toma de decisiones frente a la situación financiera planteada inicialmente.

A continuación, se podrá visualizar uno de los módulos contenidos en la Guía, usado para la descripción de esta experiencia.

\section{CONCLUSIONES}

La información obtenida instó a que se construyera una propuesta estructurada en módulos a la que se le denominó Guía, donde cada módulo conserva un énfasis de formación acorde a las necesidades que no permitían al estudiante avanzar en el proceso de formación financiera. La Guía contempla enfoques didácticos activos propuestos por la Institución Universitaria Antonio José Camacho en su Modelo Pedagógico (2013), donde se enfatiza el aprendizaje colaborativo, autónomo y significativo.
Las Tecnologías de la Información para la Comunicación son agentes de motivación para los estudiantes y el apropiarlos muestra el impacto positivo en su uso, porque el estudiante no ve desligado de estas el proceso de modelación matemática en una situación del ámbito financiero. Por esta razón, en cada módulo se da una situación para crear en Excel un simulador que modele temas como el interés simple, interés compuesto, cambio de tasas, entre otros.

Lo anterior permite "que los estudiantes puedan interactuar, interpretar, argumentar y proponer sobre situaciones reales de un concepto financiero, facilitando el acercamiento al proceso de modelación y al fortalecimiento del pensamiento numérico y variacional" (Grueso, 2017, p. 18).

La investigación abordada puso de manifiesto una serie de situaciones problema tanto en el ámbito social como en el ámbito académico en que se encuentran los estudiantes de ciencias empresariales de la UNIAJC al ingresar al curso de Matemática Financiera, las cuales no les permiten tener una adecuada comprensión de los conceptos del contexto financiero.

El diseño de la propuesta didáctica en módulos integra los elementos básicos de matemáticas con el concepto financiero a través del proceso de modelación, permitiendo al estudiante tener una representación significativa del concepto, de manera que pueda identificar elementos y correlacionarlos para dar solución a una situación financiera.

Finalmente, la construcción de simuladores en Excel se convirtió en una herramienta de gran utilidad para agilizar cálculos, interpretarlos e interactuar con los resultados en el análisis y la toma de decisiones, tanto en el ámbito financiero como en otros contextos socio económicos. 


\section{REFERENCIAS BIBLIOGRÁFICAS}

Alaniz, B., Camara, V., Mas, M., y Pagura, M. (2006). Una Investigación sobre modelación matemática en la enseñanza de matemática financiera: límites y posibilidades. Santa Fe, Argentina. Aula Universitaria, 1(8), 103-109.

Biembengut, M., y Hein, N. (2000). Modelagen matematica no ensino. Sao Paulo, Brasil. Editora Contexto.

Carazo, A., y Brey, R. (2012). Errores en el aprendizaje de las Matemáticas Financieras. Enseñanza de las ciencias, 30(2), 73-92.

D’Amore, B. (2011). Didáctica de la matemática. Bogotá, Colombia. Didácticas Magisterio.

Edel, R., y García, A. (2009a). Innovar el proceso de enseñanza-aprendizaje de las matemáticas financieras: Aplicación de la triada didáctica E-T-S. Trabajo presentado en el X congreso nacional de investigación educativa. Veracruz, México. Área 7 entornos virtuales de aprendizaje.

Edel, R., y García, A. (2009b). Un modelo didáctico basado en el diseño de simuladores: el caso de la matemática financiera. Alcances y posibilidades de incorporación de la ESAD: Experiencias más recientes en México y Centroamérica. Guanajuato, México. Revista del consejo de Ciencias y Tecnología del estado de Guanajuato, 46(4).

García, J. (2008). Matemáticas Financieras con ecuaciones de diferencia finita. Bogotá, Colombia. Pearson Prentice Hall

Grueso, E. (2017). Propuesta didáctica a través de la modelación matemática en matemática financiera para los estudiantes de ciencias empresariales de la UNIAJC (tesis de maestría). Universidad Tecnológica de Pereira e Institución Universitaria Antonio José Camacho, Cali, Colombia. Recuperado de https://core.ac.uk/download/ pdf/153513307.pdf

Institución Universitaria Antonio José Camacho. (2013). Modelo pedagógico Institucional. Cali: Institución Universitaria Antonio José Camacho

Ministerio de Educación Nacional de Colombia. (1998). Lineamientos Curriculares de Matemáticas. Bogotá, Colombia: Cooperativa Editorial Magisterio.

OECD. (2017). PISA 2015 Results (Volume IV): Students' Financial Literacy. http://dx.doi. org/10.1787/9789264270282-en

Villa, J. (2007). La modelación como proceso en el aula de matemáticas: Un marco de referencia y un ejemplo. Revista Tecno-Lógicas, 19(1), 63-85. 


\section{LOS AUTORES}

\section{Emiliano Grueso Cárdenas}

Magister en la Enseñanza de las Matemáticas de la Universidad Tecnológica de Pereira, Contador Público de la Universidad Libre Seccional Cali. Licenciado en Matemáticas de la Universidad Santiago de Cali, con experiencia docente mayor a 20 años, directivo docente por más de 7 años. Actualmente Docente Ocasional Tiempo Completo de la Institución Universitaria Antonio José Camacho, adscrito al Departamento de Ciencias Básicas y miembro del Grupo de Investigación GISCBA. Correo electrónico: egruesoc@admon. uniajc.edu.co

\section{Liliana Andrea Potosí Cruz}

Estudiante de segundo año del Doctorado en Educación de la Universidad Santiago de Cali. Magister en Educación con énfasis en Educación Matemática de la Universidad del Valle. Especialista en Gerencia Educativa con énfasis en gestión de proyectos de la Universidad Católica de Manizales. Licenciada en Matemáticas y Física de la Universidad del Valle. Desde el año 1997 al 2007 docente de matemáticas y física en la educación básica y media de instituciones privadas y oficiales de Cali y sus alrededores. Durante el periodo 2007-2008 capacitadora a nivel nacional del Modelo Educativo de la Media Académica Rural (MEMA RURAL), convenio entre la Universidad de Pamplona y el MEN. A partir del año 2009 docente de la UNIAJC. actualmente Docente Ocasional Tiempo Completo de la Institución Universitaria Antonio José Camacho, adscrita al Departamento de Ciencias Básicas y miembro del Grupo de Investigación GISCBA. Correo electrónico: lpotosi@admon.uniajc.edu.co

\section{Sandra Esther Suárez Chávez}

Magister en Enseñanza de la Matemática de la Universidad Tecnológica de Pereira (UTP) en convenio con la Institución Universitaria Antonio José Camacho (UNIAJC), Especialista en Gerencia de Proyectos de la Universidad del Tolima en convenio con la UNIAJC e Ingeniera Industrial de la Universidad Autónoma de Occidente. Docente del área de Matemáticas en la UNIAJC desde el año 2007, entre los años 2008 y 2015 fue directora del programa de Articulación Educativa de la UNIAJC, periodo en el cual diseñó y ejecutó varios proyectos con la Alcaldía de Santiago de Cali y convocatorias del Ministerio de Educación Nacional de Colombia (MEN), y publicó el libro "La Articulación de los Subsistemas". En la actualidad, es docente Ocasional de Tiempo Completo del Departamento de Ciencias Básicas de la Institución Universitaria Antonio José Camacho y miembro del Grupo de investigación GISCBA. Correo electrónico: ssuarez@admon. uniajc.edu.co 